\title{
Antibacterial and Antibiofilm Activity of Mercaptophenol Functionalized-Gold Nanorods Against a Clinical Isolate of Methicillin-Resistant Staphylococcus Aureus
}

Nahla O. Eltai

Qatar University

Nouf Mahmoud ( $\nabla$ nouf.mahmoud@zuj.edu.jo)

Al-Zaytoonah Private University of Jordan https://orcid.org/0000-0002-3870-7568

Zain Z. Zakaria

Qatar University

Hana Abdelrahman

Qatar University

Ala-Eddin Al Moustafa

Qatar University

Maha Al-Asmakh

Qatar University

\section{Research Article}

Keywords: Gold nanorods, 4-mercaptophenol, MRSA, antibiofilm, MIC, bactericidal.

Posted Date: December 22nd, 2021

DOI: https://doi.org/10.21203/rs.3.rs-1179467/v1

License: (c) (i) This work is licensed under a Creative Commons Attribution 4.0 International License.

Read Full License

Version of Record: A version of this preprint was published at Journal of Inorganic and Organometallic Polymers and Materials on April 6th, 2022. See the published version at https://doi.org/10.1007/s10904022-02294-0. 


\section{Abstract}

Gold nanorods (AuNRs) were synthesized and surface functionalized with 4-mercaptophenol (4-MPH) ligand. The surface-functionalized AuNRs, 4-MPH-AuNRs, were characterized by UV-Vis spectroscopy, dynamic light scattering (DLS), transmission electron microscopy (TEM) imaging, zeta potential, and Fourier-Transformed Infrared (FTIR) spectroscopy. The antibacterial and antibiofilm activities of 4-MPHAuNRs were evaluated against a clinical isolate of Methicillin-Resistant Staphylococcus aureus (MRSA). The results indicate that the surface-modified nanorods, 4-MPH-AuNRs, exhibit a bactericidal activity with a minimum inhibitory concentration (MIC) of $\sim 6.25 \mu \mathrm{g} / \mathrm{ml}$ against a planktonic suspension of MRSA. Furthermore, 4-MPH-AuNRs resulted in 2-3 log-cycle reduction of MRSA biofilm viable count over a concentration range of $100-4.0 \mu \mathrm{g} / \mathrm{ml}$. The bacterial uptake of surface-modified nanorods was investigated by inductively coupled plasma-optical emission spectroscopy (ICP-OES) and scanning electron microscopy (SEM) imaging; the results reveal that $27 \%$ of the nanorods were internalized into the bacterial cells after $6 \mathrm{hrs}$ of exposure. SEM imaging revealed a significant accumulation of the nanorods at the bacterial cell wall and a possible cellular internalization. Thus, 4-MPH-AuNRs can be considered a potential novel antibacterial agent, particularly against resistant MRSA strain biofilm.

\section{Introduction}

Antimicrobial resistance has become a challenge in treating bacterial infections, particularly those encountered in chronic wound infections; thus, there is substantial increasing demand for the development of new materials with promising antimicrobial activity against a wide range of infectious microbes ${ }^{12,3}$.

Nanomedicine is a well-established branch of science that uses nanodevices, such as nanoparticles, acquiring unique diagnostic, biological, biotechnological, and therapeutic properties ${ }^{4,5}$. With huge surface areas and exceptional physical and chemical properties, nanomaterials demonstrate several applications in electronics, catalysis, and medicine ${ }^{6,7}$. Recently, organic and inorganic nanomaterials have provided an alternative method to treat bacterial infectious diseases ${ }^{8}$.

Among inorganic nanoparticles, gold nanoparticles (AuNPs) present with several unique physical and chemical properties making them attractive for several biomedical applications such as sensing, imaging, diagnosis, and drug delivery and therapy 4,910 . Therefore, interest in exploiting the potential biocidal activities of AuNPs has risen recently due to their compatibility with mammalian cells, high colloidal stability, and easy conjugation to biomolecules and antibiotics ${ }^{11-15}$. The nanoparticles' size, shape, and surface chemistry have a crucial impact on their interaction with biological systems and their cellular uptake and cytotoxicity ${ }^{16-18}$. Recently, Piktel et al. compared the antibacterial and antibiofilm activities of AuNPs of different shapes; they found that gold nano-peanut and AuNRs were effective against several Escherichia coli (E. coli) strains as potential antimicrobial agents for surface coatings of medical devices ${ }^{13}$. 
Surface functionalization of AuNRs is crucial to enhance their colloidal stability and reduce the concentration of toxic surfactants involved in their synthesis; furthermore, this allows the conversion of the nanoparticle to nanotherapeutics ${ }^{19,20}$. We have demonstrated previously the antibacterial and antibiofilm activities of AuNRs functionalized with several chemical moieties against various grampositive bacterial strains ${ }^{21-23}$. Also, we presented the utilization of AuNRs as a potential drug delivery of an anti-fungal agent ${ }^{24}$.

Thiolated chemical molecules are commonly used to functionalize the surface of the AuNPs due to the strong binding affinity of gold towards thiol ${ }^{25}$. 4-mercaptophenol (4-MPH) is a small organosulfur compound that has been used in several sensing and therapeutic applications; recently, 4-MPH and thioglycolic acid-coated AuNPs were used for the detection of phytic acid and iron ions ${ }^{26}$. Furthermore, derivatives of 4-MPH were effective as novel anticancer agents against melanoma and breast cancer cell lines ${ }^{27,28}$. In general, organosulfur compounds exhibit several biological activities, particularly antimicrobial, against a broad spectrum of bacteria, fungi, and viruses ${ }^{29}$; for example, 2-mercaptoethanol showed antibacterial activity towards Propionibacterium skin culture ${ }^{30}$.

Among pathogens contributing to skin infections, Staphylococcus aureus ( $S$. aureus), a gram-positive bacterium, is the most common bacterium responsible for skin and community-acquired infections. It represents a significant burden on the healthcare system ${ }^{31}$. Methicillin-Resistant Staphylococcus aureus (MRSA) is a resistant strain of $S$. aureus, which has a biofilm-forming ability and is considered most of the adherent $S$. aureus ${ }^{32,33}$ with limited options of treatment by antibiotics ${ }^{34}$. Bacterial biofilm is an assembly of bacterial cells attached to a surface and enclosed within extracellular polymeric substances (EPS) ${ }^{35}$. Due to the complex properties of biofilms, they are resistant to environmental challenges and antibiotics that complicate and limit their treatment options ${ }^{35}$. Nanomedicine has shown promising benefits to prevent and eradicate bacterial biofilms formation via several mechanisms ${ }^{36}$. AuNPs of different surface chemistries demonstrated anti-biofilm activity against various bacterial biofilms; such as $S$. aureus and $P$. aeruginosa and other types of bacterial strains 21,37 .

In this study, surface functionalization of AuNRs with 4-MPH ligand will be performed by the surface ligand exchange method. The antibacterial and anti-biofilm activities, and bacterial uptake of the 4-MPHAuNRs is investigated for the first time against a clinical isolate of MRSA strain.

\section{Material And Methods}

\subsection{Synthesis and characterization of AuNRs 2.1.1 Synthesis of AuNRs and PEG-AuNRs}

AuNRs of the aspect ratio of $\sim 4$ were synthesized using a mixture of surfactants as described previously 38 . Synthesized nanorods were functionalized with a small concentration of methoxy-polyethylene glycol- 
thiol (m-PEG-SH, MW $\sim 5000 \mathrm{~g} \mathrm{~mol}^{-1}$, Sigma Aldrich, USA) to enhance their colloidal stability. A volume of $0.1 \mathrm{ml}$ of a $1 \mathrm{mg} \mathrm{ml}^{-1} \mathrm{~m}$-PEG-thiol solution was added to each $1.0 \mathrm{ml}$ of twice-centrifuged AuNRs and left for $6 \mathrm{hrs}$ with continuous stirring followed by centrifugation at $10000 \mathrm{rpm}$ for $10 \mathrm{~min}$. The concentrated nanorods pellets were collected and dispersed in ultrapure water.

\subsubsection{Functionalization of PEG-AuNRs with 4- Mercaptophenol (4-MPH), 4-MPH-AuNRs}

Synthesized PEG-AuNRs were functionalized with 4-MPH (Sigma Aldrich, USA) by adding an equivalent amount of $6.0 \mathrm{mg}$ of 4-MPH to each $1.0 \mathrm{ml}$ of twice-centrifuged nanorods and left overnight with continuous stirring. The surface-functionalized AuNRs solution was centrifuged at $8000 \mathrm{rpm}$ for $8 \mathrm{~min}$. Then the concentrated nanorods pellets were collected and dispersed in ultrapure water.

\subsubsection{Characterization of AuNRs and 4-MPH-AuNRs}

Synthesized AuNRs were characterized by UV-vis absorbance at 200-1100 nm (UV-1800 spectrophotometer, Shimadzu, Japan), surface charge, and hydrodynamic size (Nicomp Nano Z3000 size/zeta potential analyzer, Entegris, USA). AuNRs samples with appropriate dilution were filled into the dynamic light scattering cuvettes for hydrodynamic radius measurement, or into folded capillary cells for zeta potential measurement at $25^{\circ} \mathrm{C}$. Zeta potential of 4-MPH-AuNRs was measured upon mixing with the bacterial growth medium after 24 hrs of exposure. Mean values and standard deviations were calculated from at least three measurements. The shape of the nanoparticles was confirmed by transmission electron microscope (TEM) imaging (Morgani 268 TEM, FEl, The Netherlands); using Formvar coated TEM copper grids.

Surface functionalization of AuNRs with 4-MPH was also confirmed by Fourier-transformed infrared (FTIR) spectroscopy (Shimadzu, Japan). The AuNRs samples were freeze-dried and prepared as potassium bromide ( $\mathrm{KBr}$ ) disks for FTIR measurements.

\subsection{Antibacterial activity of 4-MPH-AuNRs against Methicillin-resistant Staphylococcus aureus (MRSA)}

\subsubsection{Bacterial strain and growth condition}

Clinical isolates of Methicillin-resistant Staphylococcus aureus (MRSA) strains were obtained from the Microbiology's bacterial culture collections at the Biomedical Research Centre (BRC), Qatar University. MRSA strains were cultured overnight on nutrient agar (Remel, ThermoFisher Scientific, Lenexa, KS, USA), incubated at $37^{\circ} \mathrm{C}$ for $24 \mathrm{hr}$, then adjusted to $0.5 \mathrm{MacFarland}$ Standard as measured by DensiCHEK PLUS (bioMérieux, France), and used throughout the study. Longstanding preservation of cultures was done at $-80^{\circ} \mathrm{C}$ in cryovial tubes (Technical Service Consultant, Lancashire, UK).

\subsubsection{Minimal inhibitory concentration (MIC)}


The antibacterial activity of AuNRs suspension was scrutinized using the standard two-fold broth microdilution method, to determine the minimal inhibitory concentration (MIC) of AuNRs against MRSA planktonic cultures, according to the Clinical and Laboratory Standards Institute (CLSI, 2020). Briefly, seven two-fold AuNRs suspension serial dilutions (100 $\mu \mathrm{l}$ each), of $100,50,25,12.5,6.25,3.12,1.5 \mu \mathrm{g} / \mathrm{ml}$ were added to the wells in a 96-well flat -bottom plate (Microtest ${ }^{\mathrm{tm}} 96$ tissue culture plate, Franklin Lakes, NJ, USA) containing $100 \mu$ LMuller Hinton Broth (MHB), (Liofilchem ${ }^{\circledR}$, Roseto degli Abruzzi, Italy). Then, $10 \mu \mathrm{L}$ of standardized 0.5 MacFarland inoculum was added to each well within $15 \mathrm{~min}$ of its preparation to achieve an inoculum of $1.5 \times 10^{6} \mathrm{CFU} /$ well in each well. The plate included MHB plus AuNRs as sterility controls and MHB plus MRSA as growth control. Finally, the inoculated 96 well plates were covered with a lid and incubated in ambient air at $37^{\circ} \mathrm{C}$ for $24 \mathrm{hr}$. MIC was recorded as the average lowest AuNRs concentration that completely inhibits MRSA growth as detected by the unaided eye (clear solutions). This experiment was replicated a minimum of three times.

\subsubsection{Minimum bactericidal concentration (MBC)}

MBC of AuNRs was determined by streaking $20 \mu$ of a tenfold serial dilution of the MIC onto nutrient agar free from AuNRs. The neutralization of the antimicrobial activity of the tested compound was achieved by dilution. The plates were then incubated at $37^{\circ} \mathrm{C}$ for 16 to $18 \mathrm{hr}$. Bacterial colonies, if any, were enumerated to determine viable $\mathrm{CFU} / \mathrm{ml}$. A comparable, controlled experiment was conducted as above, however, without the application of AuNRs. The number of bacterial colonies was used to calculate viable bacterial cells $(\mathrm{CFU} / \mathrm{ml})$. The percent reduction in the viable count and log reduction in viability were calculated as a measure of the bactericidal activity of surface-functionalized AuNRs ${ }^{23}$. In order to exclude the possible antibacterial activity of the coating material; 4-MPH, the MIC test was performed for an aqueous dispersion of 4-MPH.

\subsection{Antibiofilm activity of 4-MPH-AuNRs against Methicillin- resistant Staphylococcus aureus (MRSA) biofilm}

\subsubsection{Biofilm culture of MRSA}

Before their use, all borosilicate glass beads $3-4 \mathrm{~mm}$ in diameter (ISOLAB Laborgeräte $\mathrm{GmbH}$, Singapore) were rinsed in a soap solution, washed in $\mathrm{ddH}_{2} \mathrm{O}$, incubated overnight in $80 \%$ ethanol, and finally thoroughly cleaned in sterile water. Then, bacteria were cultivated on these beads as described previously ${ }^{21}$ to form biofilm. Concisely, each bead was placed into a well in a 96- flat microtiter plate containing $200 \mu \mathrm{L}$ of nutrient broth and $20 \mu \mathrm{L}$ of a standardized $0.5 \mathrm{McFarland}$ MRSA culture to obtain an inoculum size $1.5 \times 10^{6} \mathrm{CFU} / \mathrm{ml}$. Then the plates were incubated aerobically at $37^{\circ} \mathrm{C}$ and $100 \mathrm{rpm}$ for $72 \mathrm{hrs}$ in a shaker incubator. After every $24 \mathrm{hrs}$, the consumed media was substituted carefully by $200 \mu \mathrm{L}$ of new media. After biofilm culturing, beads were washed three times using normal saline to remove loosely attached bacterial cells. Then, biofilm densities formed at 24,48 , and 72 hrs were determined by placing the beads in an Eppendorf tube with $200 \mu$ of nutrient broth and vortexed for 1 min to release the attached bacteria. After that, $100 \mu \mathrm{l}$ of the suspension was serially diluted over ten-fold serial dilutions, 
and $20 \mu \mathrm{l}$ was streaked on nutrient agar media. The number of colonies and dilution factors were reported to determine biofilm density as CFU/bead quantitatively.

\subsubsection{Antibiofilm activity of AuNRs suspension against MRSA}

The antibiofilm activity of AuNRs suspension on 72-hr biofilm cultures was determined by challenging the washed biofilm beads with exposure to AuNRs (100, 40, 20, and $6 \mu \mathrm{g} / \mathrm{ml})$ suspension for $24 \mathrm{hrs}$ incubation at $37^{\circ} \mathrm{C}$. After treatment, beads were washed with normal saline, and viable biofilm density was determined, as previously mentioned in section 2.3.1 21 . The antibiofilm activity was estimated as a percentage reduction of viability compared to the positive control of untreated biofilm ${ }^{21}$.

\subsection{Imaging of MRSA biofilm by scanning electron microscopy (SEM)}

The biofilm on the glass bead was dip-washed in $\mathrm{ddH}_{2} \mathrm{O}$ and fixed in the previously mentioned fixative for 48 hrs. Consequently, all samples were air-dried, mounted on a stub with adhesive carbon tape, then sputter-coated with a 12-nm layer of gold and examined by SEM (ZEISS 1530 Gemini, Carl Zeiss Microscopy $\mathrm{GmbH}$, Germany) operating at $5 \mathrm{kV}$.

\subsection{Quantification of bacterial uptake of 4-MPH-AuNRs by Inductively-coupled plasma- optical emission spectrometry (ICP-OES)}

Samples were prepared in a liquid form through digestion to produce an aqueous solution in Nitric acid. Briefly, 4.0 McFarland MRSA suspended in $250 \mu \mathrm{L}$ Müller Hinton was treated with AuNRs for $6 \mathrm{hrs}$ at $37^{\circ} \mathrm{C}$ and then centrifuged for $5 \mathrm{~min}$ at $8000 \mathrm{rpm}$. After that, the formed pellet (weight $0.19-0.20 \mathrm{gm}$ ) was washed with $1 \% \mathrm{HNO}_{3}$, followed by five times deionized water. Consequently, the washed pellets were lyophilized by a vacuum freeze-drier (VirTis SP scientific, USA) for $30 \mathrm{hrs}$ condensation at $-70^{\circ} \mathrm{C}$. Afterward, $2.0 \mathrm{ml} \mathrm{HNO}_{3}$ (65\%) were added to the sample, placed on a hot block (environmental express, Charleston, South Carolina) at $105^{\circ} \mathrm{C}$ for $2 \mathrm{hrs}$, and volumed up to $10 \mathrm{ml}$ by adding distilled deionized water. The reagent blanks were treated precisely as were the samples. Then the cellular uptake of AuNRs was determined using ICP-OES (Optima, 7300 DV Perkin Elmer, South Carolina, USA).

\subsection{Imaging of bacterial uptake of 4-MPH-AuNRs by SEM}

MRSA suspension of 1.0 MacFarland $(250 \mu \mathrm{l})$ was treated with $50 \mu \mathrm{L}$ of $50 \mu \mathrm{g} / \mathrm{ml}$ for 6 hrs at $37^{\circ} \mathrm{C}$. After that, treated bacterial cells were washed with deionized water; then the pellet was fixed with $2 \%$ glutaraldehyde. Subsequently, the sample was examined using TEM (TECNAI G2 TEM, TF20, FEI, USA).

\section{Results And Discussion}




\subsection{Synthesis and characterization of AuNRs}

AuNRs were synthesized using a mixture of surfactants (oleic acid and cetyltrimethylammonium bromide: $C T A B$ ), to finetune the size uniformity and adjustability ${ }^{39}$. The synthesized nanorods were functionalized with a low concentration of PEG to enhance their colloidal stability towards next step of surface functionalization with 4-MPH. The optical spectrum of AuNRs demonstrated two distinct peaks at 529 and $880 \mathrm{~nm}$ for the longitudinal and transverse peaks, respectively (Figure 1A). Obtained surfacefunctionalized AuNRs (4-MPH-AuNRs) exhibited slightly shifted longitudinal plasmon due to the difference in the dielectric constant (Figure 1A). TEM imaging clearly showed the nanoparticles' rod shape with an average length and width of $\sim 74 \mathrm{~nm}$ and $\sim 14 \mathrm{~nm}$, respectively (Figure 1B). The average hydrodynamic size of the nanorods measured by dynamic light scattering before and after functionalization was $\sim 88 \mathrm{~nm}$ and $81 \mathrm{~nm}$, respectively (Figure C). The average surface charge of the nanorods before and after functionalization was $+4 \mathrm{mV}$ and $-23 \mathrm{mV}$, respectively (Figure 1D), which confirm the successful surface functionalization of the nanorods with 4-MPH.

4-MPH is a small thiolated compound; its assembly on the nanorods' surface has occurred through a covalent bond with gold which has a well-known high affinity towards free sulfhydryl groups ${ }^{40}$. Thiolated ligands provide higher colloidal stability to AuNPs than other ligands that bind to the nanoparticles through hydrophobic or electrostatic attractions ${ }^{40}$. Slight broadening of the longitudinal peak of 4-MPHAuNRs may be related to the possible binding of the nanorods to 4-MPH through the O-AuNRs bond. A previous study showed such binding induced aggregation of the AuNPs at $\mathrm{pH} \sim 10{ }^{41}$; however, the $\mathrm{pH}$ of the 4-MPH-AuNRs in our study is between 6-7.

FTIR spectroscopy was used to confirm the surface functionalization of the nanoparticles with 4-MPH. As presented in Figure 2, 4-MPH and 4-MPH-AuNRs demonstrated very similar FTIR spectra; both have broadband at $\sim 3400 \mathrm{~cm}^{-1}$ which corresponds to $-\mathrm{OH}$ stretching and sharp band at $\sim 1200 \mathrm{~cm}^{-1}$ corresponds to $\mathrm{C}-\mathrm{H}$ bending in the benzene ring. Furthermore, 4-MPH showed a weak band at $\sim 2560$ $\mathrm{cm}^{-1}$ that corresponds to $-\mathrm{SH}$ stretching; fading of this band in the spectrum of 4-MPH-AuNRs suggests successful surface functionalization of the nanorods via S-Au covalent bond ${ }^{17}$.

\subsection{Antibacterial activity of 4-MPH-AuNRs against MRSA 3.2.1 Minimal inhibitory and biocidal concentrations; MIC and MBC}

Minimum inhibitory concentration (MIC) is defined as the lowest concentration of a compound or antimicrobial agent that inhibits the visible growth of a specific microorganism, whereas minimum bactericidal concentration (MBC) is the lowest concentration of a compound or antimicrobial agent showing no growth after exposure. MIC and MBC are commonly used in in-vitro studies to evaluate new compounds' antibacterial activity and to reveal bacterial resistance ${ }^{42}$. 
Our results indicate that the MIC of 4-MPH-AuNRs is estimated to be $6.0 \mu \mathrm{g} / \mathrm{ml}$ (Figure 3). Interestingly, the MBC of 4-MPH-AuNRs was the same as the MIC, indicating that the nanorods in our study have bactericidal activity towards this strain of MRSA. Bactericidal antibiotics actively kill the bacteria compared to bacteriostatic agents that slow or inhibit the growth of bacteria but do not kill them; generally, bactericidal agents have a ratio of $\mathrm{MBC}$ to $\mathrm{MIC} \leq 4{ }^{43}$.

AuNPs have shown a broad spectrum of antibacterial activity against Gram-positive and Gram-negative bacteria ${ }^{44}$. In addition to their chemical and physical merits, AuNRs do not develop drug resistance easily compared to conventional antibacterial agents, which is considered an interesting advantage, particularly against resistant and biofilm-producing pathogens 44,45 .

The size and shape of AuNRs have a crucial role in their biomedical responses ${ }^{46,47}$; for example, nanoflowers and nanostars were shown to demonstrate considerable antibacterial activity against $S$. aureus ${ }^{48}$; Furthermore, nanoclusters exhibited promising antibacterial activity against different types of multidrug-resistant bacteria in a previous study ${ }^{49}$.

On the other hand, the surface chemistry of nanoparticles has significant effects on their antibacterial activity. AuNPs conjugated to antibiotics or chemical ligands have demonstrated appreciable antibacterial activities towards a wide spectrum of Gram-positive and Gram-negative bacteria; for example, the antibacterial activity of AuNPs functionalized with Amoxicillin ${ }^{50}$, enzyme-like activity compounds ${ }^{51}$, chitosan ${ }^{52}$ or supported on mesoporous silica ${ }^{53}$ has been displayed against $S$. aureus and other bacterial strains. We previously demonstrated that AuNRs coated with a phospholipid moiety demonstrated low MIC value and high antibacterial activity compared to AuNRs coated with a cholesterol moiety against $S$. aureus strain ${ }^{22}$.

In this study, we demonstrated for the first time the antibacterial activity of AuNRs coated with 4-MPH ligand against MRSA, a resistant strain with a biofilm-forming ability. Generally, nanoparticles show preferential binding to Gram-positive pathogens, such as Listeria monocytogenes, $S$. aureus, or Streptococcus pyrogens, with enhanced antibacterial activity ${ }^{54}$. 4-MPH-AuNRs exhibited a low MIC value compared to AuNRs coated with other surface chemistries ${ }^{21,22}$. 4-MPH-AuNRs are easily synthesized and exhibit good colloidal stability.

It is worth mentioning that surface functionalization of nanoparticles, in general, determines their biocorona composition upon interaction with biological systems, consequently affecting their biological responses, cellular uptake, and toxicity ${ }^{55}$. Although the 4-MPH-AuNRs have negative-charged surface chemistry, this does not explain their bacterial uptake or biological responses since nanoparticles acquire bio-corona once exposed to biological conditions ${ }^{54,56}$. More specifically, the surface charge of 4-MPHAuNRs was increased once exposed to the bacterial growth medium due to the formation of bio-corona around the nanoparticles, which determines their various biological responses such as cytotoxicity and 
cellular uptake. A recent study revealed that biomolecules could drastically affect the inhibitory effect of the nanomaterials under physiological conditions ${ }^{54}$.

\subsection{Antibiofilm activity of 4-MPH-AuNRs against MRSA biofilm}

\subsubsection{Biofilm formation}

The antibiofilm activity of surface-functionalized nanorods 4-MPG-AuNRs was investigated at the MIC concentration and higher. The MRSA biofilm was grown on the surface of beads, and the formed biofilm was characterized by quantifying the number of bacteria/bead at different time points (Figure 4) and SEM imaging (Figure 5).

The bacterial CFU/bead was found to be $6.1 \times 10^{7}, 7.4 \times 10^{8}$, and $4.12 \times 10^{9}$ after 24,48 , and 72 hrs of culturing onto the glass beads, respectively, at $37^{\circ} \mathrm{C}$ (Figure 4). The SEM images reveal the formation and attachment of the biofilm on the surface of glass beads and biofilm density formed onto the surface at each time point (Figure 5A-D), where the highest density was obtained after $72 \mathrm{hrs}$ of bacterial culturing (Figure 5D).

Adherence and attachment of bacteria cells to form a biofilm on a surface is dependent on many factors, such as environmental conditions, types, and surface properties of materials. Glass and other polymeric surfaces are commonly used to enhance the formation of bacterial biofilms, which produce extracellular materials that facilitate attachment and matrix formation ${ }^{35}$.

\subsubsection{Anti-biofilm activity of 4-MPH-AuNRs against MRSA biofilm}

The inhibition activity of the nanorods on the bacterial biofilm was estimated by calculating the percentage reduction and log-cycle reduction of the biofilm upon treatment with four different concentrations of 4-MPH-AuNRs. As described in Table 2, 4-MPH-AuNRs resulted in a 2-3 log-cycle reduction of the viable bacterial count of the MRSA biofilm. 
Table 2

Percentage reduction and log-cycle reduction of viable biofilm after treatment with 4-MPH-AuNRs of different concentrations for $24 \mathrm{hrs}$.

\begin{tabular}{|llll|}
\hline 4-MPH-AuNRs $(\mu \mathrm{g} / \mathrm{ml})$ & $\begin{array}{l}\text { Conc of bacteria post-treatment } \\
(\mathrm{CFU} / \mathrm{ml})\end{array}$ & $\%$ Reduction of viable count & $\begin{array}{l}\text { Log-cycle } \\
\text { reduction }\end{array}$ \\
\hline 100 & $5 \times 10^{6}$ & 99.88 & 2.9 \\
\hline 40.0 & $4.4 \times 10^{7}$ & 98.93 & 1.97 \\
\hline 20.0 & $6.5 \times 10^{7}$ & 98.42 & 1.8 \\
\hline 6.0 & $6.3 \times 10^{7}$ & 98.47 & 1.81 \\
\hline
\end{tabular}

Biofilm formation of bacteria is considered one of the essential virulence factors that enhances the pathogen's colonization and infections, particularly in wound infections ${ }^{57,58}$. Biofilm is a complex structure of adherent bacterial that proliferate and accumulate extracellular matrix ${ }^{58}$. Eradication of bacterial biofilms is considered a challenge for conventional antibiotics. Various previous reports demonstrate the ability of AuNPs to inhibit or eradicate the bacterial biofilm; for example, AuNPs coated with polyethylene glycol/polyethyleneimine strongly inhibited biofilm formation of Candida albicans and 57 , in addition, we demonstrated previously the ability of surface-functionalized AnNRs to eradicate Pseudomonas aeruginosa biofilm by the photothermal mechanism of action ${ }^{21}$. In order to inhibit or eradicate the bacterial biofilm, nanoparticles should penetrate the bacterial biofilm and disrupt its structure; penetration into the biofilm depends on many factors such as size, shape, and surface chemistry of the nanoparticles and the biofilm characteristics and maturity ${ }^{59}$. The observed significant eradication of the biofilm by 4-MPH-AuNRs indicates the ability of the nanorods to penetrate efficiently into the MRSA biofilm, disrupt and eradicate its matrix. We hypothesize that such an effect could be related to mechanical deformation of the biofilm resulting from accumulation and adsorption of the nanoparticles at the biofilm matrix and bacterial cell membrane ${ }^{60}$.

\subsection{Cellular uptake of AuNRs into MRSA by ICP-OES and SEM imaging}

In order to understand the interaction of 4-MPH-AuNRs with MRSA and their bacterial uptake, we quantified the percentage of bacterial uptake of the nanorods by the ICP-OES method. The results indicate that the average bacterial uptake of AuNRs inside the cell was $\sim 30 \mu \mathrm{g} / \mathrm{g}$ after $6 \mathrm{hrs}$ of exposure, corresponding to $\sim 27 \%$ of the total amount of applied nanorods. The results demonstrate that an appreciable amount of the nanorods were internalized into bacterial cells and consequently enhanced their effect. SEM imaging of treated bacteria reveal that the nanorods were accumulated at the surface of the bacterial cells with possible internalization into the bacterial cell wall after 6 hrs of incubation, and eventually, cellular rupture and death. Cellular uptake of nanoparticles is essential for their biological 
responses and toxicity; nanoparticles have been conjugated with specific ligands to intensify their internalization into bacterial cells and consequently, their antibacterial activity ${ }^{61}$.

In addition to the crucial role of bio-corona in modulating the nanoparticles' cellular uptake and antibacterial mechanism of action, the nanoparticles' size plays a crucial role too. A recent study demonstrated that the antibacterial mechanism of action of nanoparticles within the size range of $80-$ $100 \mathrm{~nm}$ is caused by mechanical deformation of the bacterial cell membrane due to their adsorption at the cellular membrane ${ }^{60,62}$. On the other hand, the antibacterial activity of smaller nanoparticles is related to their translocation through the bacterial cell membrane and forming irreversible pores ${ }^{63}$.

\section{Conclusions}

Gold nanoparticles provide promising solutions to address challenges related to bacterial resistance and decreased efficiency. 4-MPH functionalized-gold nanorods exhibited potent antibacterial and antibiofilm activities towards a clinical isolate of MRSA. The surface-functionalized nanorods exhibited considerable internalization into the bacterial cells, which may justify their cytotoxicity against the bacterial biofilm. 4$\mathrm{MPH}-A u N R s$ is a potential nano-system to eradicate planktonic and biofilm of MRSA, particularly for topical chronic wounds.

\section{Declarations}

\section{Acknowledgments}

The authors thank the Deanship of Scientific Research at Al-Zaytoonah University of Jordan (20202019/12/28) and Qatar University (BRC-2021-ID-02, QUST-2-CHS-2021-2019) for the financial funding.

\section{Disclosures}

The authors declare no conflict of interest.

\section{References}

1. M. Balouiri, M. Sadiki and S. K. Ibnsouda, Journal of Pharmaceutical Analysis, 2016, 6, 71-79.

2. N. Durán, M. Durán, M. B. de Jesus, A. B. Seabra, W. J. Fávaro and G. Nakazato, Nanomedicine: Nanotechnology, Biology and Medicine, 2016, 12, 789-799.

3. R. Jayakumar, M. Prabaharan, P. T. Sudheesh Kumar, S. V. Nair and H. Tamura, Biotechnology Advances, 2011, 29, 322-337.

4. S. Bayda, M. Adeel, T. Tuccinardi, M. Cordani and F. Rizzolio, Molecules, 2019, 25. 
5. L. Gonzalez, R. J. Loza, K.-Y. Han, S. Sunoqrot, C. Cunningham, P. Purta, J. Drake, S. Jain, S. Hong and J.-H. Chang, Journal of Ocular Pharmacology and Therapeutics, 2013, 29, 124-134.

6. P. Dallas, V. K. Sharma and R. Zboril, Advances in Colloid and Interface Science, 2011, 166, 119-135.

7. H.-J. Park, S. Park, J. Roh, S. Kim, K. Choi, J. Yi, Y. Kim and J. Yoon, Journal of Industrial and Engineering Chemistry, 2013, 19, 614-619.

8. R. Kumar, A. Umar, G. Kumar and H. S. Nalwa, Ceramics International, 2017, 43, 3940-3961.

9. M. Saeedi, M. Eslamifar, K. Khezri and S. M. Dizaj, Biomed Pharmacother, 2019, 111, 666-675.

10. Nouf N. Mahmoud, D. A. Sabbah, R. Abu-Dahab, D. Abuarqoub, M. Abdallah, Ameerah and E. A. Khalil, RSC Advances, 2019, 9, 12718-12731.

11. E. E. Connor, J. Mwamuka, A. Gole, C. J. Murphy and M. D. Wyatt, Small, 2005, 1, 325-327.

12. Pradeepa, S. M. Vidya, S. Mutalik, K. Udaya Bhat, P. Huilgol and K. Avadhani, Life Sci, 2016, 153, 171179.

13. E. Piktel, Ł. Suprewicz, J. Depciuch, S. Chmielewska, K. Skłodowski, T. Daniluk, G. Król, P. KołatBrodecka, P. Bijak, A. Pajor-Świerzy, K. Fiedoruk, M. Parlinska-Wojtan and R. Bucki, Scientific Reports, $2021,11,12546$.

14. M. M. Abdel-Kareem and A. A. Zohri, Letters in Applied Microbiology, 2018, 67, 465-475.

15. Y. Zhao, Y. Tian, Y. Cui, W. Liu, W. Ma and X. Jiang, Journal of the American Chemical Society, 2010, $132,12349-12356$.

16. T. Chatterjee, B. K. Chatterjee and P. Chakrabarti, Sci Rep, 2017, 7, 9671.

17. N. N. Mahmoud, R. Abu-Dahab, L. A. Hamadneh, D. Abuarqoub, H. Jafar and E. A. Khalil, Molecular Pharmaceutics, 2019, 16, 4149-4164.

18. N. N. Mahmoud, A. Albasha, S. Hikmat, L. Hamadneh, R. Zaza, Z. Shraideh and E. A. Khalil, Biomaterials Science, 2020, 8, 1669-1682.

19. J. R. Nicol, D. Dixon and J. A. Coulter, Nanomedicine (Lond), 2015, 10, 1315-1326.

20. G. Sanità, B. Carrese and A. Lamberti, Frontiers in Molecular Biosciences, $2020,7$.

21. A. G. Al-Bakri and N. N. Mahmoud, Molecules, 2019, 24.

22. N. N. Mahmoud, A. A. Alhusban, J. I. Ali, A. G. Al-Bakri, R. Hamed and E. A. Khalil, Scientific Reports, $2019,9,5796$.

23. N. N. Mahmoud, A. M. Alkilany, E. A. Khalil and A. G. Al-Bakri, Scientific Reports, 2018, 8, 6881.

24. K. M. Hamad, N. N. Mahmoud, S. Al-Dabash, L. A. Al-Samad, M. Abdallah and A. G. Al-Bakri, RSC Advances, 2020, 10, 25889-25897.

25. V. Spampinato, M. A. Parracino, R. La Spina, F. Rossi and G. Ceccone, Frontiers in chemistry, 2016, 4, 8-8.

26. K. Koç Ö, A. Üzer and R. Apak, Mikrochim Acta, 2020, 187, 586.

27. P. Ruzza, A. Rosato, A. Nassi, M. Rondina, M. Zorzin, C. R. Rossi, M. Floreani and L. Quintieri, J Med Chem, 2009, 52, 4973-4976. 
28. D. B. Shpakovsky, C. N. Banti, E. M. Mukhatova, Y. A. Gracheva, V. P. Osipova, N. T. Berberova, D. V. Albov, T. A. Antonenko, L. A. Aslanov, E. R. Milaeva and S. K. Hadjikakou, Dalton Trans, 2014, 43, 6880-6890.

29. O. Sagdic and F. Tornuk, in Dietary Phytochemicals and Microbes, ed. A. K. Patra, Springer Netherlands, Dordrecht, 2012, DOI: 10.1007/978-94-007-3926-0_4, pp. 127-156.

30. K. L. Mattern, C. A. Evans and J. C. Lara, Appl Environ Microbiol, 1979, 37, 177-179.

31. P. Del Giudice, Acta Derm Venereol, 2020, 100, adv00110.

32. K. A. Rodvold and K. W. McConeghy, Clin Infect Dis, 2014, 58 Suppl 1, S20-27.

33. G. F. Gad, M. A. El-Feky, M. S. El-Rehewy, M. A. Hassan, H. Abolella and R. M. El-Baky, J Infect Dev Ctries, 2009, 3, 342-351.

34. P. D. Stapleton and P. W. Taylor, Sci Prog, 2002, 85, 57-72.

35. J.-S. Lee, Y.-M. Bae, S.-Y. Lee and S.-Y. Lee, Journal of Food Science, 2015, 80, M2279-M2286.

36. W. Xiu, J. Shan, K. Yang, H. Xiao, L. Yuwen and L. Wang, VIEW, 2021, 2, 20200065.

37. B. Malaekeh-Nikouei, B. S. Fazly Bazzaz, E. Mirhadi, A. S. Tajani and B. Khameneh, Journal of Drug Delivery Science and Technology, 2020, 60, 101880.

38. N. N. Mahmoud, H. Qabooq, S. Alsotari, O. A. Tarawneh, N. H. Aboalhaija, S. Shraim, A. M. Alkilany, E. A. Khalil and R. Abu-Dahab, RSC Advances, 2021, 11, 19956-19966.

39. X. Ye, C. Zheng, J. Chen, Y. Gao and C. B. Murray, Nano Lett, 2013, 13, 765-771.

40. M. H. Jazayeri, H. Amani, A. A. Pourfatollah, H. Pazoki-Toroudi and B. Sedighimoghaddam, Sensing and Bio-Sensing Research, 2016, 9, 17-22.

41. B. L. Scott and K. T. Carron, The Journal of Physical Chemistry C, 2016, 120, 20905-20913.

42. J. M. Andrews, J Antimicrob Chemother, 2001, 48 Suppl 1, 5-16.

43. G. A. Pankey and L. D. Sabath, Clin Infect Dis, 2004, 38, 864-870.

44. X. Gu, Z. Xu, L. Gu, H. Xu, F. Han, B. Chen and X. Pan, Environmental Chemistry Letters, 2021, 19, 167187.

45. X. Zhao, Y. Jia, J. Li, R. Dong, J. Zhang, C. Ma, H. Wang, Y. Rui and X. Jiang, ACS Applied Materials \& Interfaces, 2018, 10, 29398-29406.

46. Y. C. Dong, M. Hajfathalian, P. S. N. Maidment, J. C. Hsu, P. C. Naha, S. Si-Mohamed, M. Breuilly, J. Kim, P. Chhour, P. Douek, H. I. Litt and D. P. Cormode, Scientific Reports, 2019, 9, 14912.

47. X. Xie, J. Liao, X. Shao, Q. Li and Y. Lin, Scientific Reports, 2017, 7, 3827.

48. J. Penders, M. Stolzoff, D. J. Hickey, M. Andersson and T. J. Webster, Int J Nanomedicine, 2017, 12, 2457-2468.

49. Y. Li, J. Zhen, Q. Tian, C. Shen, L. Zhang, K. Yang and L. Shang, J Colloid Interface Sci, 2020, 569, 235-243.

50. S. Kalita, R. Kandimalla, K. K. Sharma, A. C. Kataki, M. Deka and J. Kotoky, Mater Sci Eng C Mater Biol Appl, 2016, 61, 720-727. 
51. W. He, Y. T. Zhou, W. G. Wamer, X. Hu, X. Wu, Z. Zheng, M. D. Boudreau and J. J. Yin, Biomaterials, 2013, 34, 765-773.

52. K. Saravanakumar, A. V. A. Mariadoss, A. Sathiyaseelan and M.-H. Wang, International Journal of Biological Macromolecules, 2020, 165, 747-757.

53. Y. Tao, E. Ju, J. Ren and X. Qu, Adv Mater, 2015, 27, 1097-1104.

54. S. Siemer, D. Westmeier, M. Barz, J. Eckrich, D. Wünsch, C. Seckert, C. Thyssen, O. Schilling, M. Hasenberg, C. Pang, D. Docter, S. K. Knauer, R. H. Stauber and S. Strieth, Biomaterials, 2019, 192, 551559.

55. P. Chandran, J. E. Riviere and N. A. Monteiro-Riviere, Nanotoxicology, 2017, 11, 507-519.

56. G. Caracciolo, O. C. Farokhzad and M. Mahmoudi, Trends in Biotechnology, 2017, 35, 257-264.

57. Q. Yu, J. Li, Y. Zhang, Y. Wang, L. Liu and M. Li, Scientific Reports, 2016, 6, 26667.

58. S. C. Davis, C. Ricotti, A. Cazzaniga, E. Welsh, W. H. Eaglstein and P. M. Mertz, Wound Repair and Regeneration, 2008, 16, 23-29.

59. A. S. Joshi, P. Singh and I. Mijakovic, Int J Mol Sci, 2020, 21.

60. D. P. Linklater, V. A. Baulin, X. Le Guével, J. B. Fleury, E. Hanssen, T. H. P. Nguyen, S. Juodkazis, G. Bryant, R. J. Crawford, P. Stoodley and E. P. Ivanova, Adv Mater, 2020, 32, e2005679.

61. M. Kumar, W. Tegge, N. Wangoo, R. Jain and R. K. Sharma, Biophysical Chemistry, 2018, 237, 38-46.

62. M. Okkeh, N. Bloise, E. Restivo, L. De Vita, P. Pallavicini and L. Visai, Nanomaterials (Basel), 2021, 11.

63. E. A. Ortiz-Benítez, N. Velázquez-Guadarrama, N. V. Durán Figueroa, H. Quezada and J. J. OlivaresTrejo, Metallomics, 2019, 11, 1265-1276.

\section{Figures}



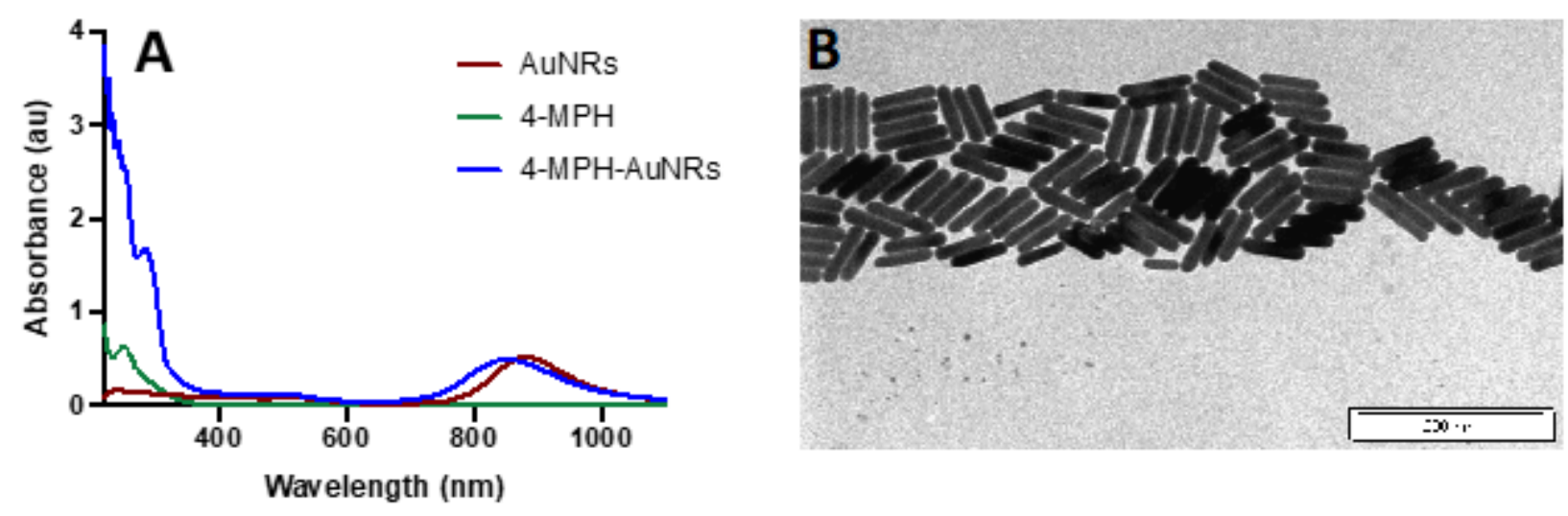

C

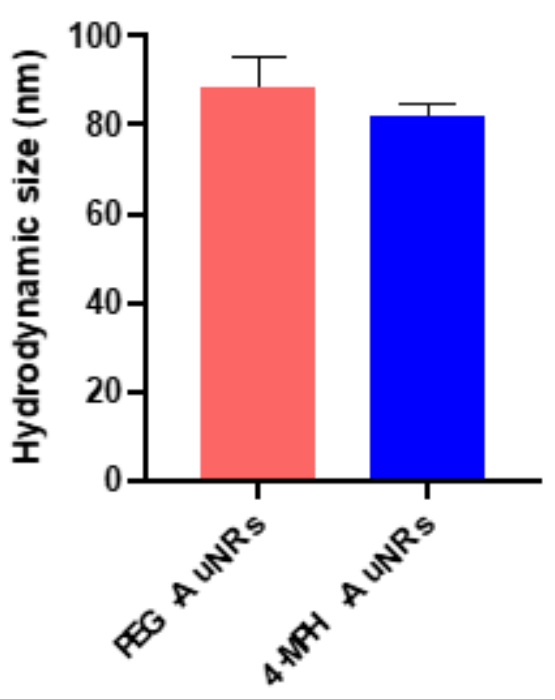

D

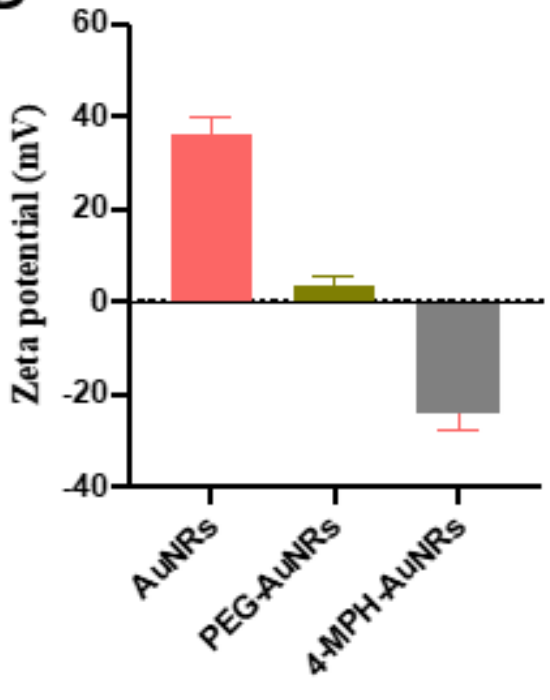

Figure 1

(A) UV-vis spectra of AuNRs, 4-MPH-AuNRs, and 4-MPH. (B) TEM image of 4-MPH-AuNRs indicates the rod shape of the nanoparticles with an average length and width of $\sim 74 \mathrm{~nm}$ and $14 \mathrm{~nm}$, respectively. (C) Hydrodynamic size of AuNRs before and after functionalization with 4-MPH. (D) Surface charge of AuNRs before and after surface functionalization with 4-MPH. 


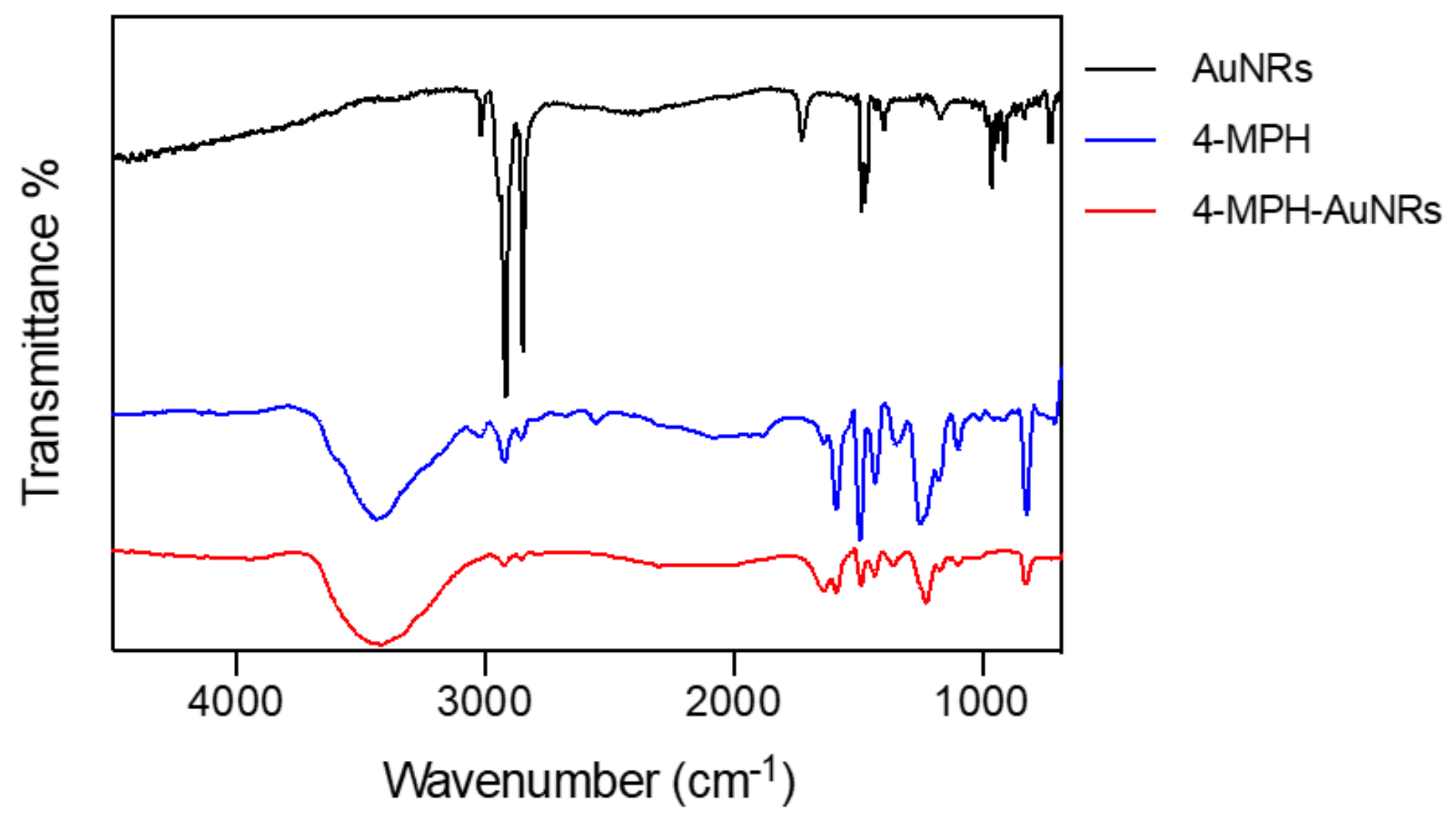

Figure 2

FTIR spectra of AuNRs, 4-MPH, and 4-MPH-AuNRs. 4-MPH and 4-MPH-AuNRs demonstrated similar spectra; both have broadband at $\sim 3400 \mathrm{~cm}^{-1}$ which corresponds to $-\mathrm{OH}$ stretching. Fading of the $-\mathrm{SH}$ band at $\sim 2560 \mathrm{~cm}^{-1}$ in the spectrum of 4-MPH-AuNRs suggests a successful functionalization through thiol-gold binding. 


\section{$100,50,25,12.5,6.25,3.12,1.5$ प}

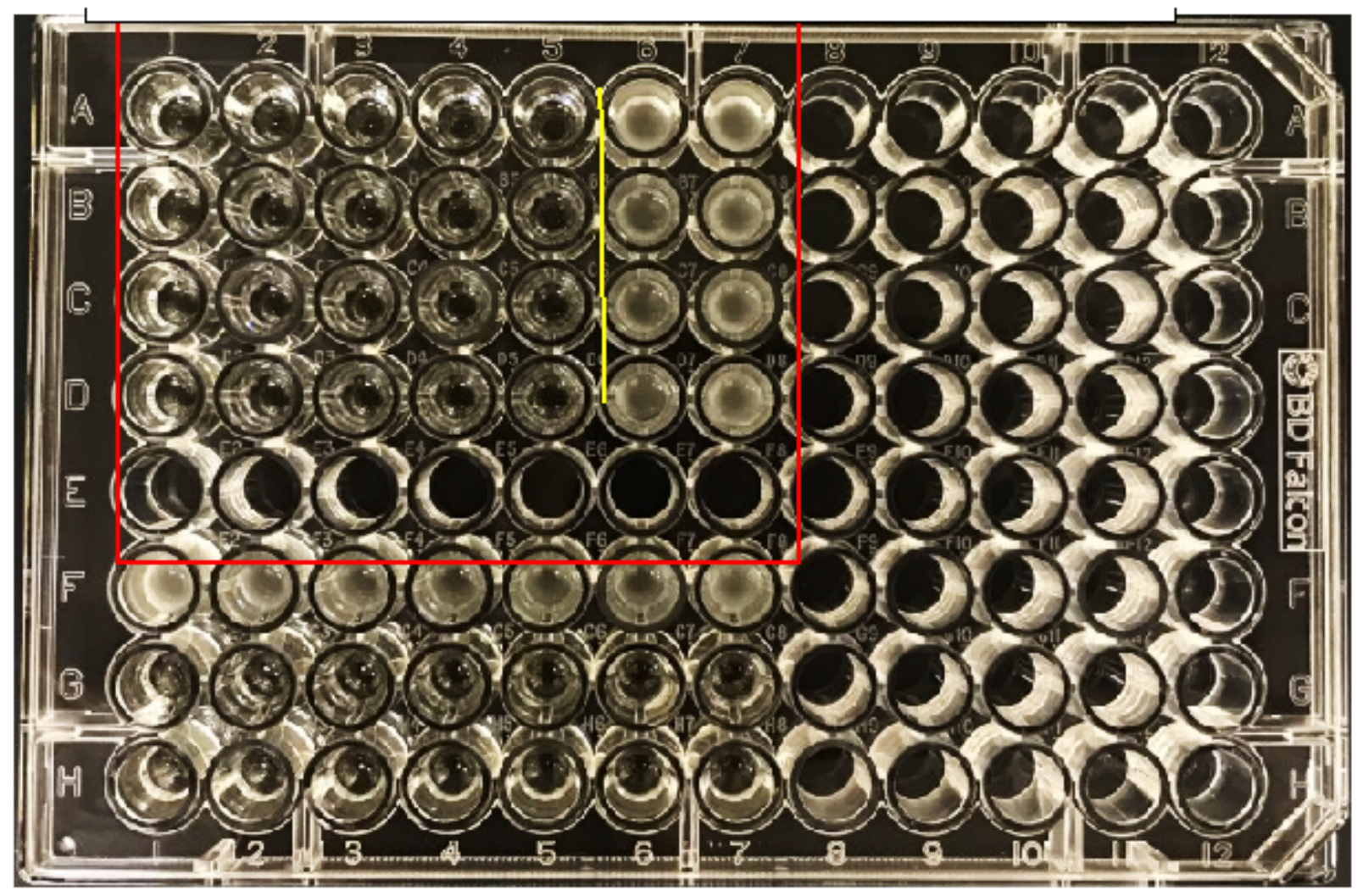

\section{Figure 3}

MIC test in 96-well plate indicates the MIC value of 4-MPH-AuNRs against MRSA. The figure displays the activity of different concentrations in double fold serial dilutions $(100,50,25,12.5,6.25,3.12,1.5 \mu \mathrm{g} / \mathrm{ml})$ of 4-MPH-AuNRs against MRSA after $24 \mathrm{hrs}$ incubation in the medium. The rows A-D are for the treatment; the concentration to the left of the yellow line indicates the MIC. F: positive growth control, G: medium sterility control, $\mathrm{H}$ : 4-MPH-AuNRs in the medium sterility control. 


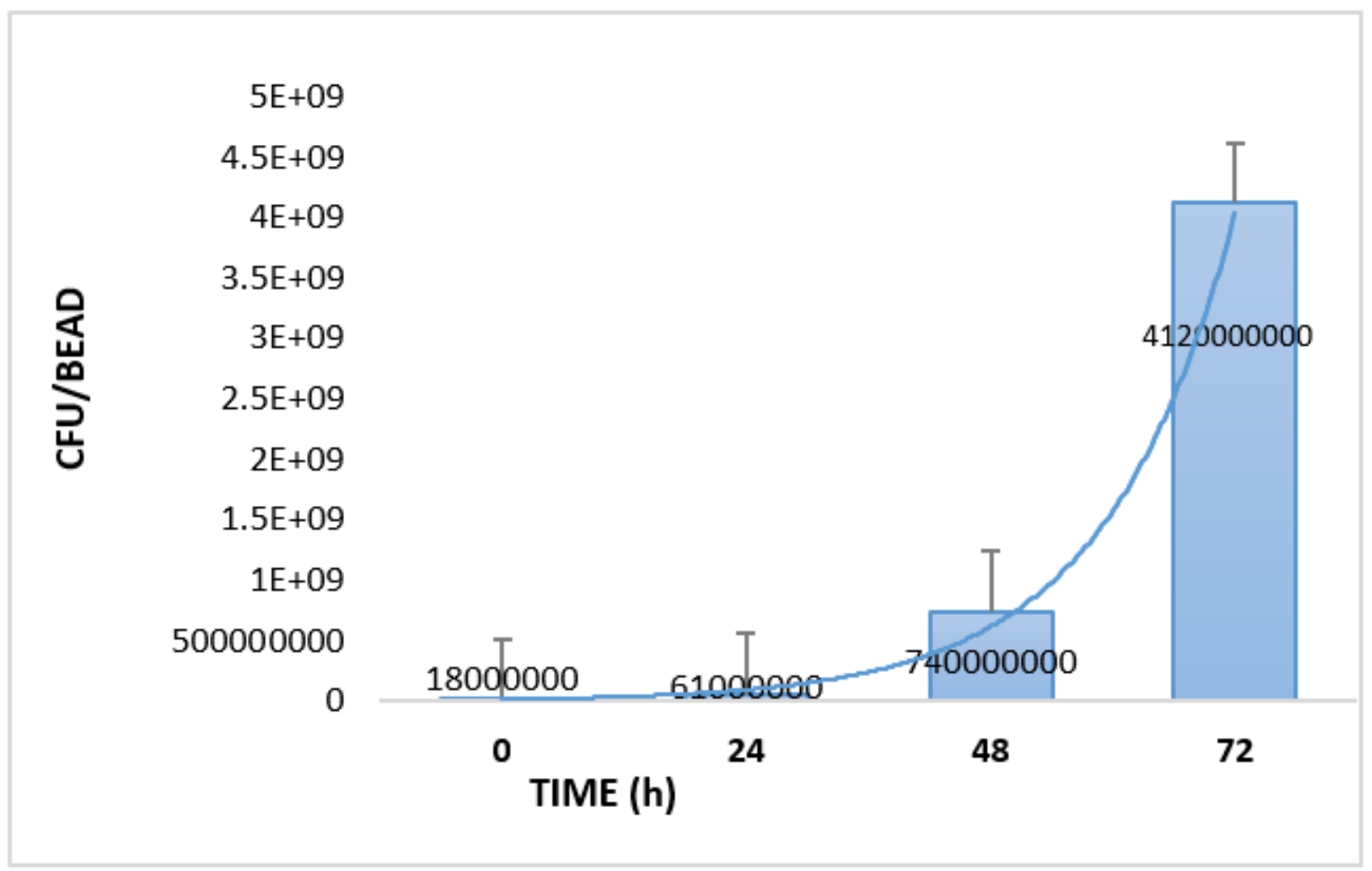

Figure 4

Number of bacteria in the biofilm formed on glass beads at $0,24,48$, and 72 hrs post- incubation. 

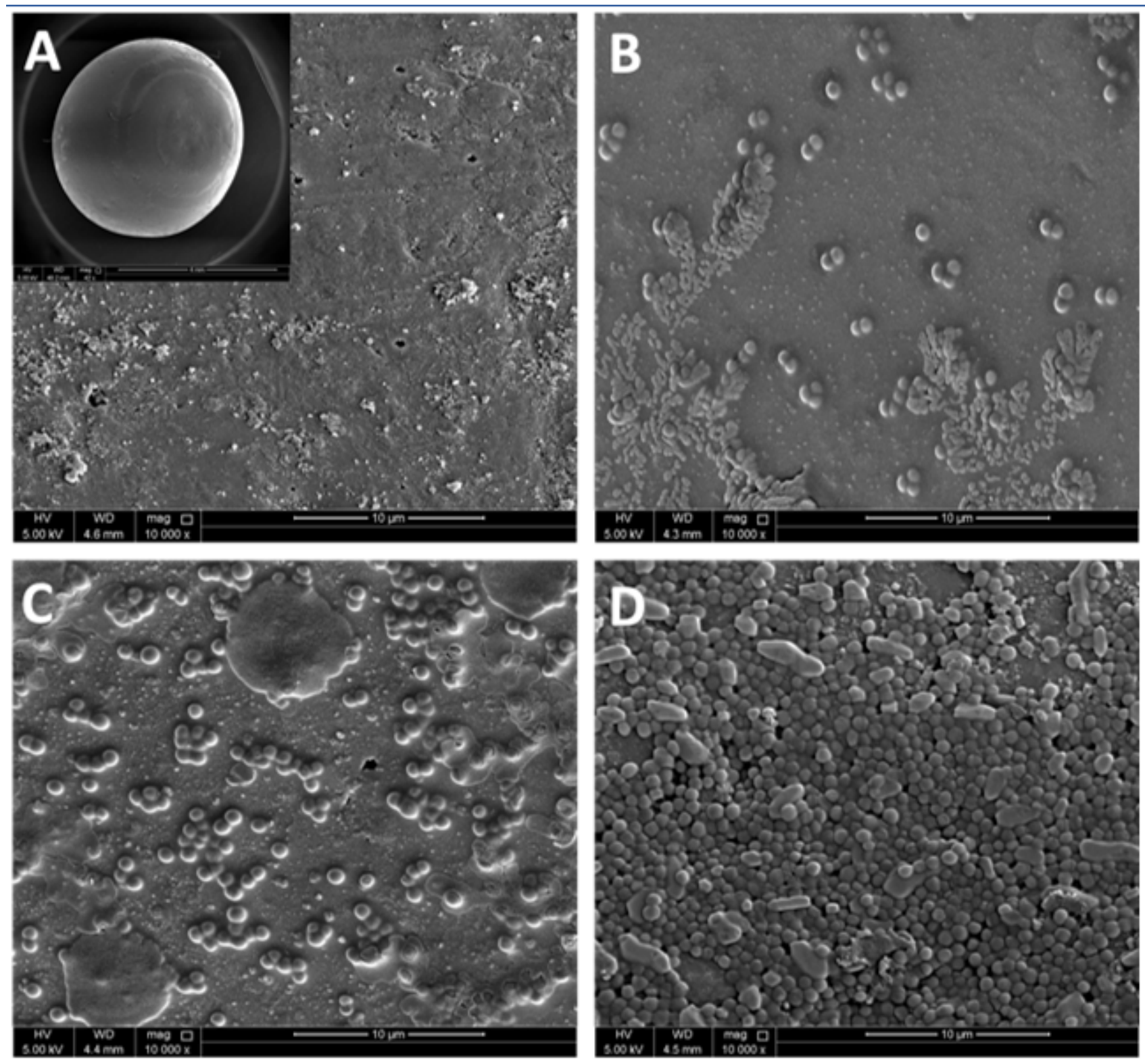

Figure 5

SEM images of the glass bead before biofilm culturing of MRSA (A), and after $24 \mathrm{hrs}(\mathrm{B}), 48 \mathrm{hrs}(\mathrm{C})$, and $72 \mathrm{hrs}(\mathrm{D})$ of biofilm culturing onto the glass beads. The high density of bacterial biofilm was observed after 72 hrs of bacterial culturing onto the glass beads. 

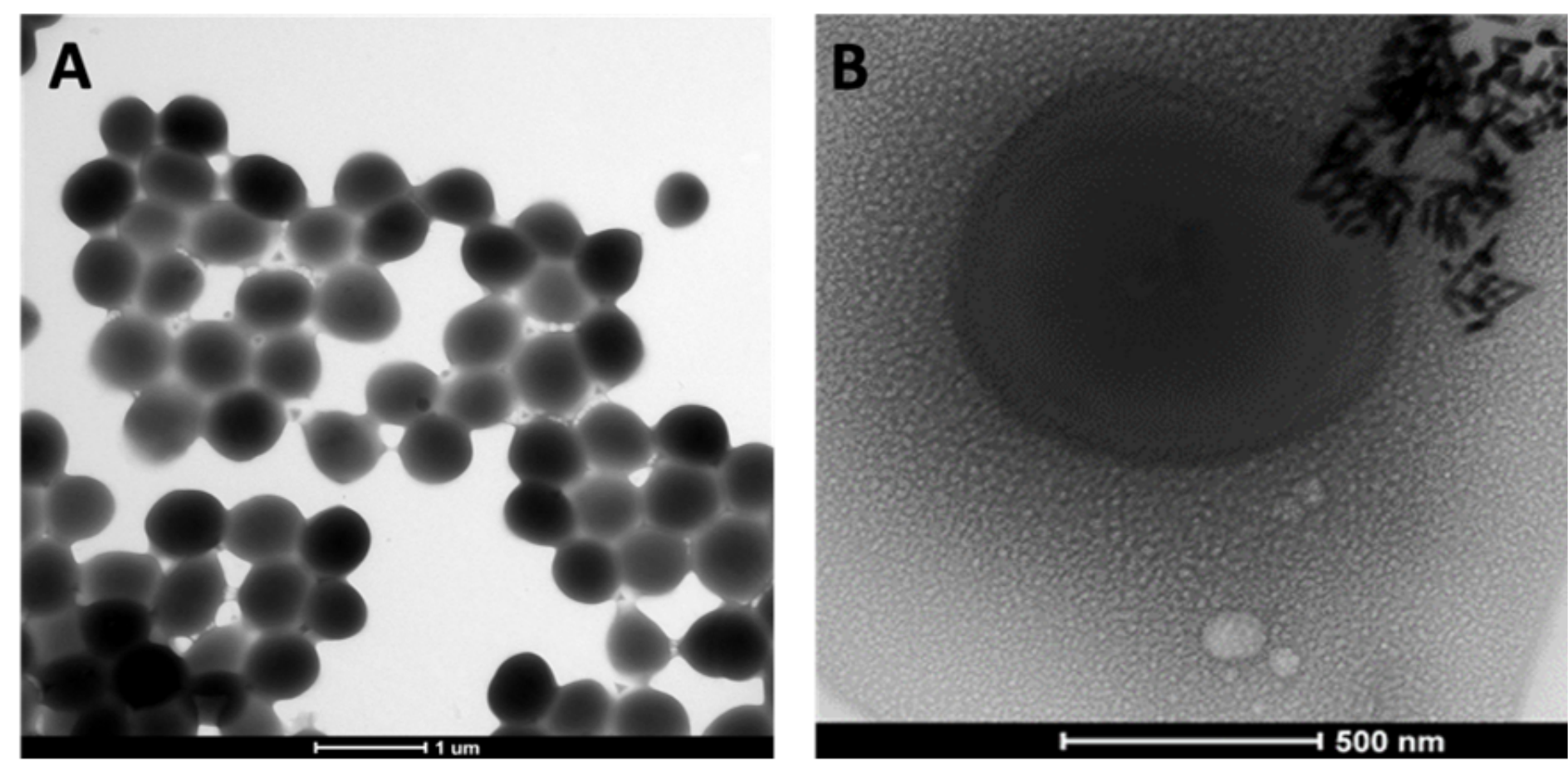

Figure 6

SEM image of $S$. aureus before (A) and after (B) treatment with 4-MPH-AuNRs for 6 hrs. The image shows the accumulation of the nanorods at the bacterial cell membrane with possible internalization across the bacterial cellular membrane resulting in mechanical deformation and cellular rupture. 